\title{
Scanning Holography Using a Modulated Linear Pupil: Simulations
}

\author{
Abdallah Mohamed Hamed \\ Physics Department, Faculty of Science, Ain Shams University, Cairo, Egypt \\ E-mail:amhamed73@hotmail.com \\ Received March 14, 2011; revised April 14, 2011; accepted April 25, 2011
}

\begin{abstract}
In this paper an electron microscopic image of diameter $80-120 \mathrm{~nm}$ and of dimensions $180 \times 220$ pixels is used as a test object to fabricate Fresnel zone plate hologram. The author proposes a different set of pupils: one pupil still being a delta function and the other being a function of $\langle\rho\rangle=\operatorname{sqrt}\left(x^{2}+y^{2}\right)$. The obtained reconstructed images in case of scanning holography are investigated.
\end{abstract}

Keywords: Scanning Holography, Linear Apertures

\section{Introduction}

The pioneer work of digital holography or computer-generated hologram $(\mathrm{CGH})$ was early proposed by Goodman, et al. [1] and Lohmann, et al. [2], and numerical hologram reconstruction was initiated by Kronrod et al. [3] in the early 1970 followed by many other authors. Improved reconstructed images from $\mathrm{CGH}$ are obtained using an iterative operations [4]. Recently, the possibilities of reconstructing the hologram structure and image from a digitally recorded specklegram without reference beam has been considered separately by Hamed [5] and Gorbatenko, et al. [6]. Also, improved reconstructed image from digital Fourier holograms is attained using superposition of reconstructed images obtained by multiple wavelengths [7] and separately using a two-step only quadratic phase shifting holography [8] where neither the reference-wave intensity nor an object-wave intensity measurement is needed in this technique.

The idea of holographic recording accomplished by heterodyne scanning was originally proposed by Poon [9-11]. And heterodyne scanning was accomplished using a twopupil optical system Lohmann and Rhodes [12]. They realized Fresnel-zone-plate-type impulse response, i.e. its phase is a quadratic function of $\mathrm{x}$ and $\mathrm{y}$, in and out-of focus plane near the focal plane of lenses $\mathrm{L}_{1}$ and $\mathrm{L}_{2}$. In a precedent proposed work by Poon, one of the pupils is a delta function and the other has a constant uniform circular aperture.

The original idea, which was later analyzed and called scanning holography [13], is to scan the 3-D object in a 2 -D raster with a complex Fresnel zone plate-type im- pulse response created by interference of a point source and a plane wave emerging from each pupil. A temporal frequency offset is introduced between the two pupils and the desired signal from a spatially integrating detector is obtained using a heterodyne detection.

In the present study, the author investigates scanning holographic imaging based on two-pupil heterodyne detection. In the original standard system proposed by Poon, one of the pupils is a delta function and the other a constant.

In the present case, the author proposes a different set of pupils: one pupil still being a delta function and the other being a function of $\rho=\operatorname{sqrt}\left(x^{2}+y^{2}\right)$. The simulated reconstructed images using the above technique of heterodyne detection are investigated. The proposal of the linearly modulated aperture [5] was investigated in a re- cent article of modulated speckle images.

\section{Theoretical Analysis}

\subsection{A Two-Pupil Heterodyne Scanning Hologram}

The optical scanning hologram is based on two-pupil heterodyne detection as shown in Figure 1. In this study, the $1^{\text {st }}$ pupil is chosen to be a linear function distributed within the circular frame of diameter $D=2 \varrho_{0}$.

$$
P_{1}(x, y)=\varrho ; \quad\left|\varrho / \varrho_{0}\right| \leq 1 \text { for linear aperture }
$$

The $2^{\text {nd }}$ pupil remains as before a delta function which is represented as follows: 


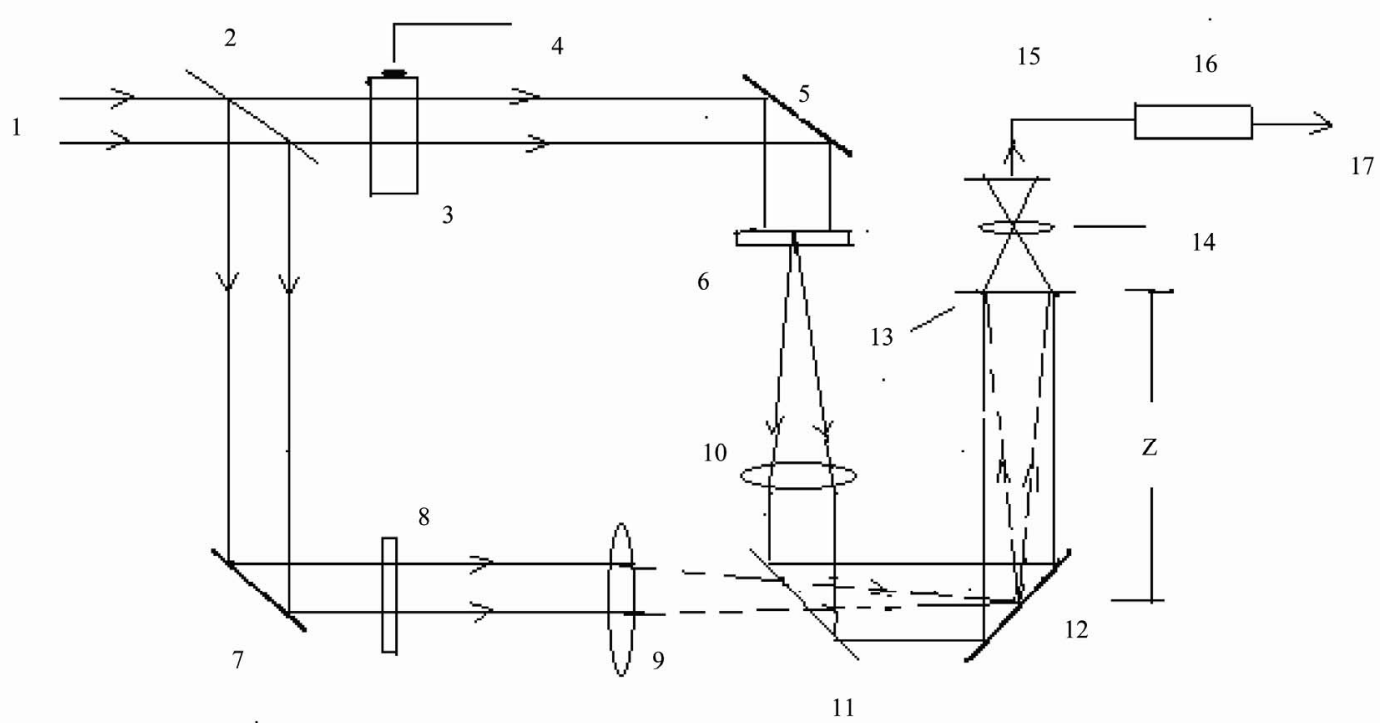

Figure 1. A two-pupil optical heterodyne scanning system. 1-Laser operating at frequency $\omega_{0} ; 2,11-b e a m$ splitters; 3acousto-optic frequency shifter; 4-cos $\Omega \mathrm{t}$ giving a modulated frequency signal at $\omega_{0}+\Omega$; 5,7 -reflecting mirrors; 2,5,7,11-form the Mach-Zehnder interferometer; 6,8-are two-pupils one pupil being a delta function and the other being a linear function of $\rho$; 9,10-are two converging lenses where the two pupils are located at the front focal planes of lenses $L_{1}$ and $L_{2}$, both with a focal length of f; 12-two dimensional scanning mirror; 13-object transparency; 14-Collector lens; 15-photo-detector; 16-electronic band pass filter tuned at the heterodyne frequency $\Omega ; 17$-output of scanned and processed current $i_{\Omega}(x, y)$.

$$
P_{2}(x, y)=\delta(x, y)
$$

The Fourier transform of Equation (1) is previously computed as follows [14]:

$$
P_{1}(k)=\text { const. }\left[\frac{J_{1}(k)}{k}+\frac{J_{0}(k)}{k^{2}}-\frac{2}{k^{2}} \sum_{i} J_{i}(k)\right]
$$

$J_{0}, J_{1}$ are Bessel functions of zero and first orders.

The optical transfer function is obtained as [10]:

$$
\begin{aligned}
& \text { OTF }\left(k_{x}, k_{y} ; z\right) \\
& \quad=\exp \left[\frac{j z}{2 k_{0}}\left(k_{x}^{2}+k_{y}^{2}\right)\right] \iint P_{1}^{*}\left(x^{\prime}, y^{\prime}\right) P_{2}\left(x^{\prime}+\frac{f}{k_{0}} k_{x}, y^{\prime}\right. \\
& \left.+\frac{f}{k_{0}} k_{y}\right) \exp \left[j \frac{z}{f}\left(x^{\prime} k_{x}+y^{\prime} k_{y}\right)\right] \mathrm{d} x^{\prime} \mathrm{d} y^{\prime}
\end{aligned}
$$

In the present work, we have assumed linear function for the $1^{\text {st }}$ pupil and the same delta function for the $2^{\text {nd }}$ pupil is used, hence substitute from (1) and (2) in Equation (4), we can write the OTF as follows:

$$
\begin{aligned}
& \text { OTF }\left(k_{x}, k_{y} ; z\right) \\
& \quad=\exp \left[\frac{j z}{2 k_{0}}\left(k_{x}^{2}+k_{y}^{2}\right)\right] \iint \sqrt{x^{\prime 2}+y^{\prime 2}} \delta\left(x^{\prime}+\frac{f}{k_{0}} k_{x}, y^{\prime}\right. \\
& \left.\quad+\frac{f}{k_{0}} k_{y}\right) \exp \left[j \frac{z}{f}\left(x^{\prime} k_{x}+y^{\prime} k_{y}\right)\right] \mathrm{d} x^{\prime} \mathrm{d} y^{\prime}
\end{aligned}
$$

This equation can be rewritten symbolically as follows:

$$
\begin{aligned}
& \operatorname{OTF}\left(k_{x}, k_{y} ; z\right)=\exp \left[\frac{j z}{2 k_{0}}\left(k_{x}^{2}+k_{y}^{2}\right)\right] F . T . \\
& \left\{\sqrt{x^{\prime 2}+y^{\prime 2}} \delta\left(x^{\prime}+\frac{f}{k_{0}} k_{x}, y^{\prime}+\frac{f}{k_{0}} k_{y}\right)\right\}
\end{aligned}
$$

Since the Fourier transform of multiplication product is transformed into a convolution product of the Fourier spectrum of each function [15], then Equation (6) becomes

$$
\begin{aligned}
\text { OTF }\left(k_{x}, k_{y} ; z\right) & =\exp \left[\frac{j z}{2 k_{0}}\left(k_{x}^{2}+k_{y}^{2}\right)\right] F . T .\left\{\sqrt{x^{\prime 2}+y^{\prime 2}}\right\} \\
& * F . T .\left\{\delta\left(x^{\prime}+\frac{f}{k_{0}} k_{x}, y^{\prime}+\frac{f}{k_{0}} k_{y}\right)\right\}
\end{aligned}
$$

The Fourier transform of a shifted delta function is calculated to give this result:

$$
F . T\left\{\delta\left(x^{\prime}+\frac{f}{k_{0}} k_{x}, y^{\prime}+\frac{f}{k_{0}} k_{y}\right)\right\}=\exp \left[-\frac{j z}{k_{0}}\left(k_{x}^{2}+k_{y}^{2}\right)\right]
$$

Substitute from Equation (8) to Equation (7), we obtain:

$$
\begin{aligned}
& \operatorname{OTF}\left(k_{x}, k_{y} ; z\right)=\exp \left[\frac{j z}{2 k_{0}}\left(k_{x}^{2}+k_{y}^{2}\right)\right] \\
& \left\{F . T .\left\{\sqrt{x^{\prime 2}+y^{\prime 2}}\right\} * \exp \left[-\frac{j z}{k_{0}}\left(k_{x}^{2}+k_{y}^{2}\right)\right]\right\}
\end{aligned}
$$

It is shown that the F.T. of the linear function $p_{1}(x, y)=$ $\rho=\left(x^{2}+y^{2}\right)^{1 / 2}$ appeared in Equation (9) is obtained in Equation (3). 


\subsection{Special case (Poon results):}

In case of uniform circular aperture to represent the $1^{\text {st }}$ pupil instead of the linear aperture, then the F.T. becomes:

$$
F . T .\left\{\sqrt{x^{\prime 2}+y^{\prime 2}}\right\}=F . T .\{1\}=\delta\left(k_{x}, k_{y}\right)
$$

In this case, Equation (9) is reduced to

$$
\begin{aligned}
& O T F_{\Omega}\left(k_{x}, k_{y} ; z\right)=\exp \left[\frac{j z}{2 k_{0}}\left(k_{x}^{2}+k_{y}^{2}\right)\right] \\
& \left\{\delta\left(k_{x}, k_{y}\right) * \exp \left[-\frac{j z}{k_{0}}\left(k_{x}^{2}+k_{y}^{2}\right)\right]\right\}
\end{aligned}
$$

From the properties of convolution product of a function with a delta function leave it unchanged, then Equation (11) is reduced to the OTF of Poon [13] to give:

$$
\mathrm{OTF}_{\Omega}\left(k_{x}, k_{y} ; z\right)=\exp \left[-\frac{j z}{2 k_{0}}\left(k_{x}^{2}+k_{y}^{2}\right)\right] ; \text { Poon's result (12) }
$$

The intensity distribution of the complex optical scanning hologram, obtained in case of uniform circular aperture for the $1^{\text {st }}$ pupil and delta function for the $2^{\text {nd }}$ pupil, is represented as :

$$
\begin{aligned}
& H_{c \pm}(x, y)=H_{\cos }(x, y) \pm j H_{\text {sin }}(x, y) \\
& \quad=\int_{D}\left\{\left|\Gamma_{0}(x, y ; z)\right|^{2} \frac{* j k_{0}}{2 \pi z} \exp \left[\frac{ \pm j k_{0}\left(x^{2}+y^{2}\right)}{2 z}\right]\right\} \mathrm{d} z
\end{aligned}
$$

Where

$$
\begin{aligned}
& H_{\cos }(x, y)=\int_{D}\left\{\left|\Gamma_{0}(x, y ; z)\right|^{2} * \frac{k_{0}}{2 \pi z} \cos \left[\frac{k_{0}\left(x^{2}+y^{2}\right)}{2 z}\right]\right\} \mathrm{d} z \\
& H_{\text {sin }}(x, y)=\int_{D}\left\{\left|\Gamma_{0}(x, y ; z)\right|^{2} * \frac{k_{0}}{2 \pi z} \sin \left[\frac{k_{0}\left(x^{2}+y^{2}\right)}{2 z}\right]\right\} \mathrm{d} z
\end{aligned}
$$

While in case of the linear pupil combined with the delta function for the $2^{\text {nd }}$ pupil, the intensity distribution of the complex optical scanning hologram is written as follows:

$$
\begin{aligned}
& H_{c \pm}(x, y)=\int_{D}\left\{\left|\Gamma_{0}(x, y ; z)\right|^{2} * \frac{j k_{0}}{2 \pi z} \exp \left[\frac{ \pm j k_{0}\left(x^{2}+y^{2}\right)}{2 z}\right] *\right. \\
& \left.\left[\sqrt{x^{2}+y^{2}} \delta\left(x+\frac{f}{k_{0}} k_{x}, y+\frac{f}{k_{0}} k_{y}\right)\right]\right\} \mathrm{d} z
\end{aligned}
$$

\section{Results and Discussion}

The original image of dimensions $180 \times 220$ pixels is plotted as shown in Figure 2. The actual dimension of the image range is $80-120 \mathrm{~nm}$. The auto-correlation intensity of the image is shown in Figure 3.

A cosine FZP hologram obtained using a linear aperture and a pinhole aperture is shown in Figure 4. The reconstructed images are obtained by operating the Fourier transform upon the holographic images which are plotted as in Figure 5(a)-(f). The reconstruction from the complex holographic FZP images are shown as in Figure 5(e) for constant pupil and in Figure 5(f) for linear pupil. It is shown that the reconstructed images from the complex holograms are much better in resolution than the reconstructed images obtained from cosine and sine holograms. Also, it is shown that the reconstructed FZP image obtained in case of linear modulation for the $1^{\text {st }}$ pupil as in Figure 5(b) is better in resolution than the reconstructed image obtained from the sine FZP hologram using circular uniform pupil Figure 5(a). This improvement in image resolution is attributed due to the resolution improvement occurred for apodized linear pupils $[5,14]$ as compared with the constant uniform circular pupils. The image profile of the original image of H1N1 virus is shown as in Figure 6(a) while the image profile of the sine FZP reconstructed image, is shown in Figure 6(b) and the image profile of the sine FZP reconstructed image using linear amplitude aperture is shown in Figure 6(c). Also, image profile of the cosine FZP reconstructed image is represented in Figure 6(d), image profile of the cosine FZP reconstructed image using linear amplitude modulation is shown in Figure 6(e), image profile of the complex FZP reconstructed image is shown in Figure 6(f), and the image profile of the complex FZP reconstructed image using linear amplitude modulation is shown in Figure 6(g). All image profiles represented in Figures 6(a)-(g) are taken at slice $x=[12,127,575]$ and slice $y=[1,180,100,100]$.

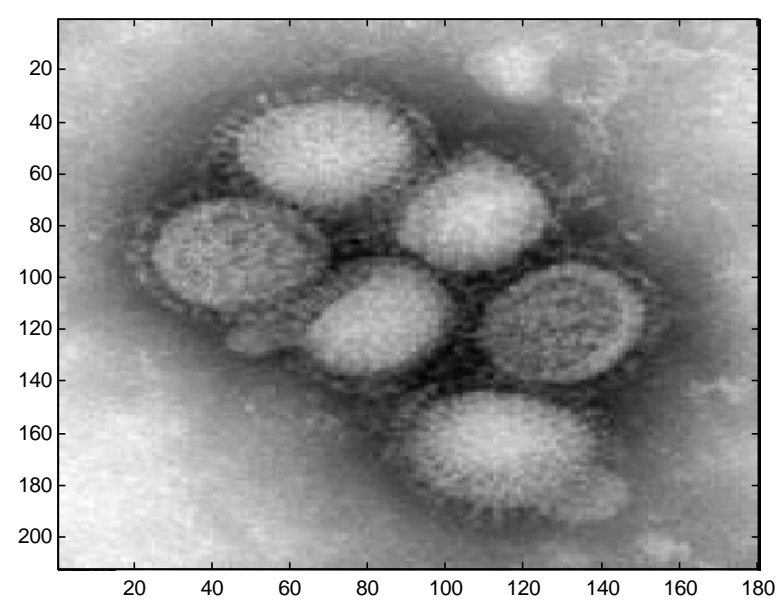

Figure 2. Electron microscope image of the reasserted $\mathbf{H}_{1} \mathbf{N}_{1}$ influenza virus photographed at the CDC influenza Laboratory. The viruses are $80-120 \mathrm{~nm}$ in diameter. The image has dimensions of $180 \times 220$ pixels. 


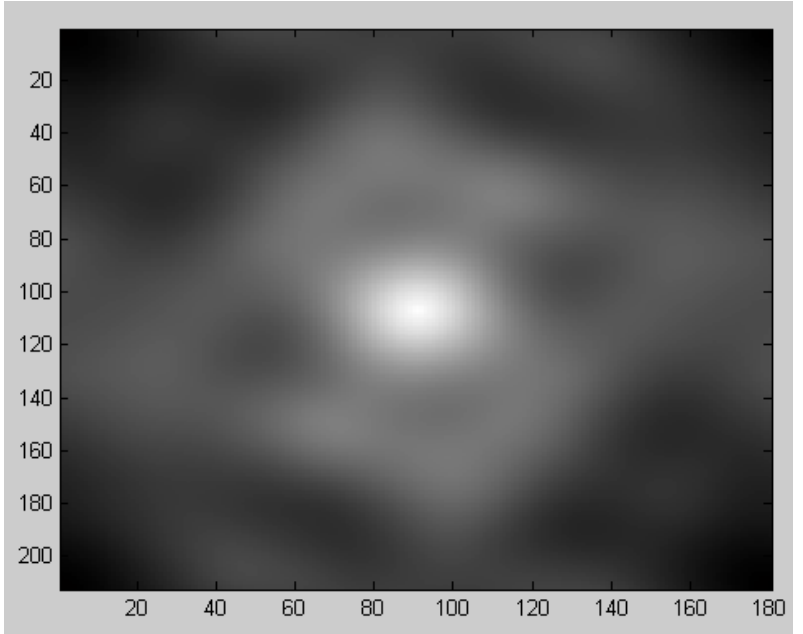

Figure 3. The auto-correlation intensity of the $H_{1} N_{1}$ image shown in Figure 2. The correlation image has dimensions of $180 \times 220$ pixels.

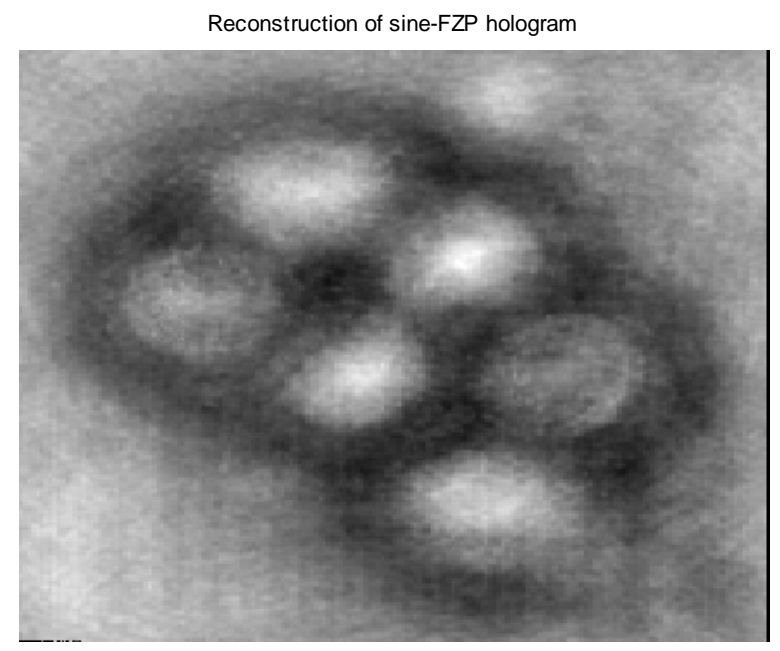

(a)

Reconstruction of cosine-FZP hologram

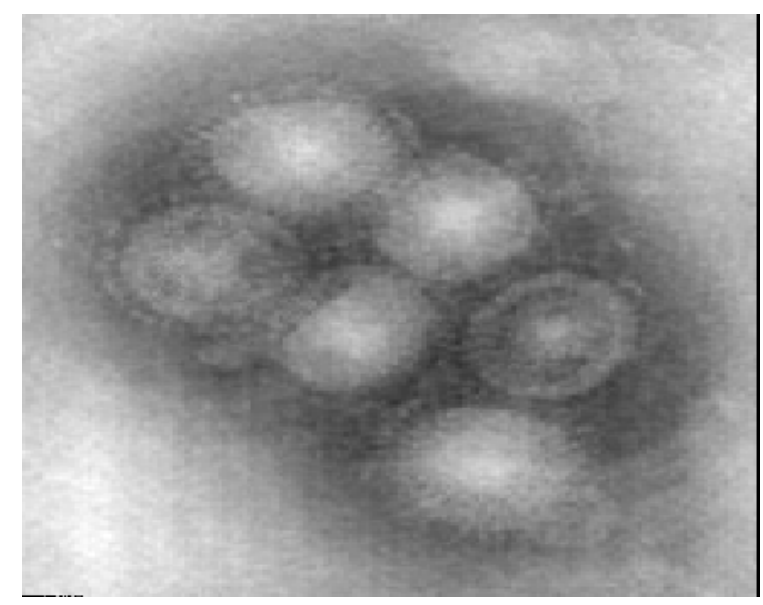

(c)

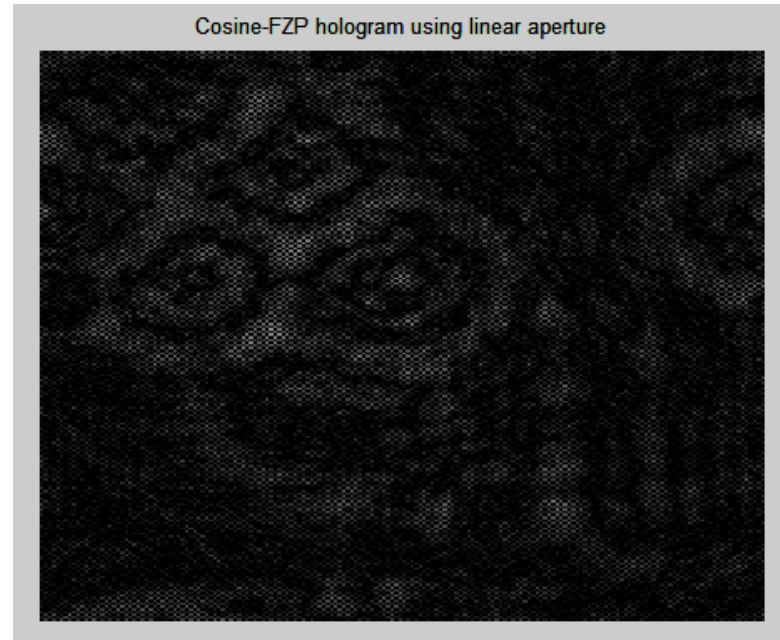

Figure 4. A cosine FZP hologram using two pupil model with one of linear distribution while the other remains the same pinhole aperture.

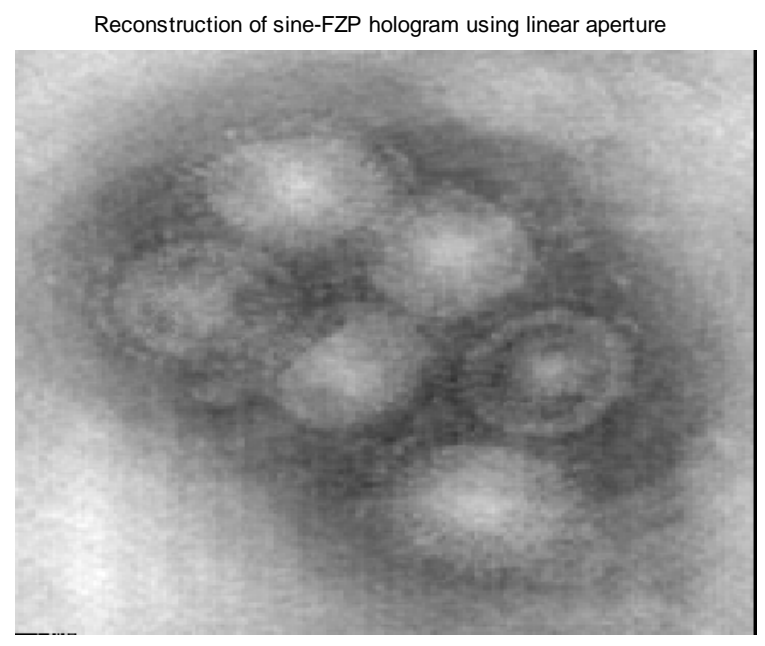

(b)

Reconstruction of cosine-FZP hologram using linear aperture

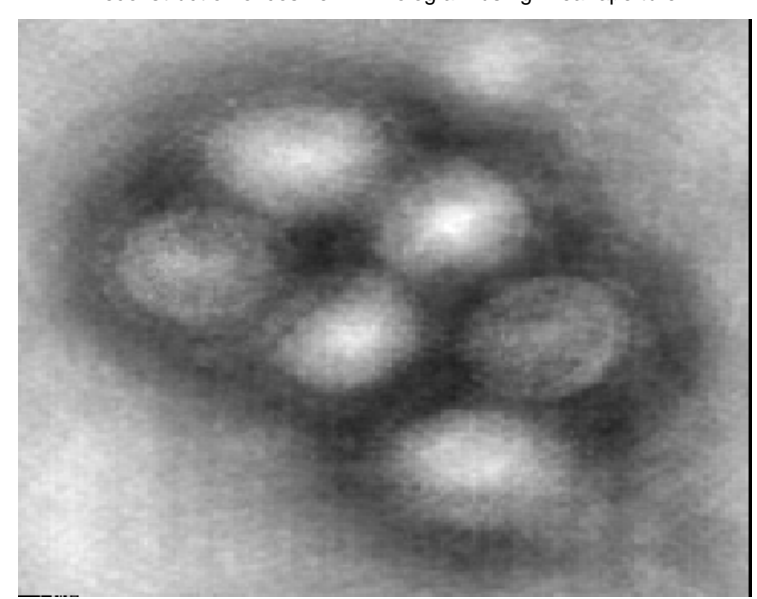

(d) 


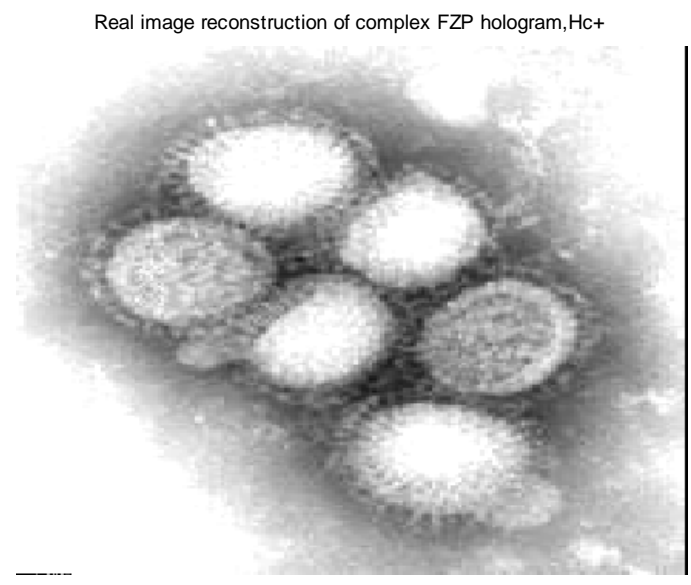

(e)

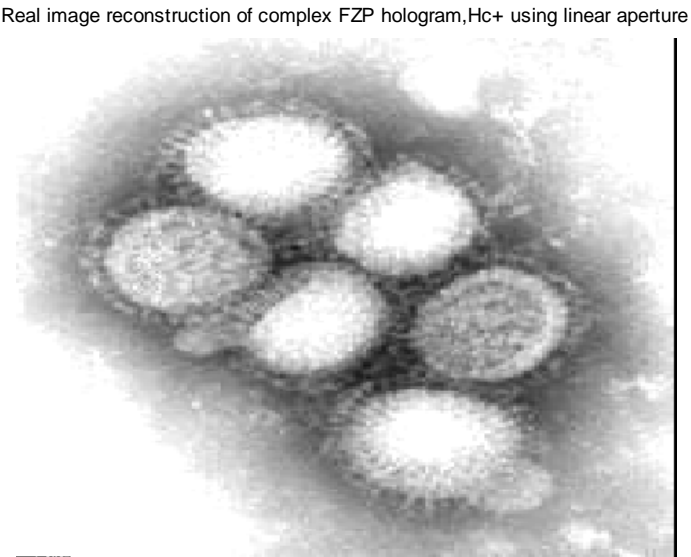

(f)

Figure 5. (a) Reconstruction of the sine-FZP hologram computed using two-pupils heterodyne detection, where the $1^{\text {st }}$ pupil is uniform circular and the $2^{\text {nd }}$ is a delta function; (b) Reconstruction of the sine-FZP hologram computed using two-pupils heterodyne detection, where the $1^{\text {st }}$ pupil is linearly distributed while the $2^{\text {nd }}$ remains a unchanged (delta function); (c) Reconstruction of the cosine-FZP hologram computed using two- pupils heterodyne detection, where the $1^{\text {st }}$ pupil is uniform circular and the $2^{\text {nd }}$ is a delta function; (d) Reconstruction of the cosine-FZP hologram computed using two-pupils heterodyne detection, where the $1^{\text {st }}$ pupil is linearly distributed while the $2^{\text {nd }}$ remains a unchanged (delta function); (e) Reconstruction of the complex-FZP hologram computed using two-pupils heterodyne detection, where the $1^{\text {st }}$ pupil is uniform circular and the $2^{\text {nd }}$ is a delta function; (f) Reconstruction of the complex-FZP hologram computed using two-pupils heterodyne detection, where the $1^{\text {st }}$ pupil is linearly distributed while the $2^{\text {nd }}$ remains a unchanged (delta function).

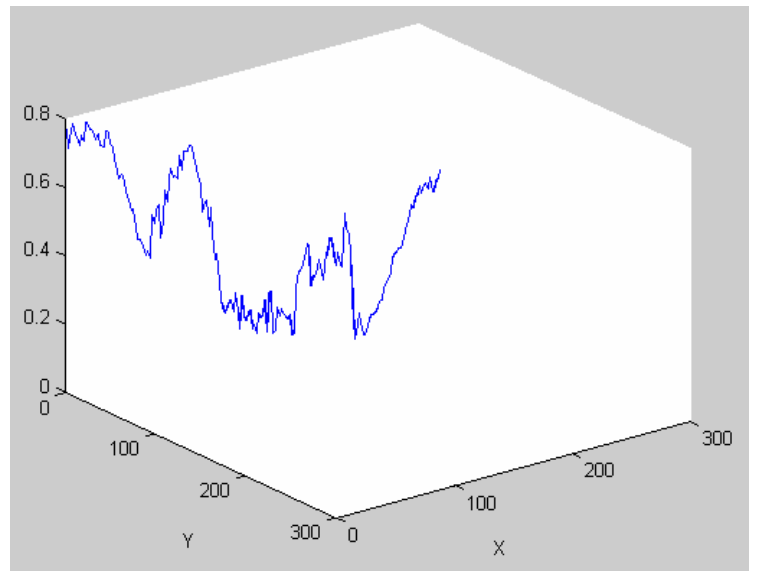

(a)

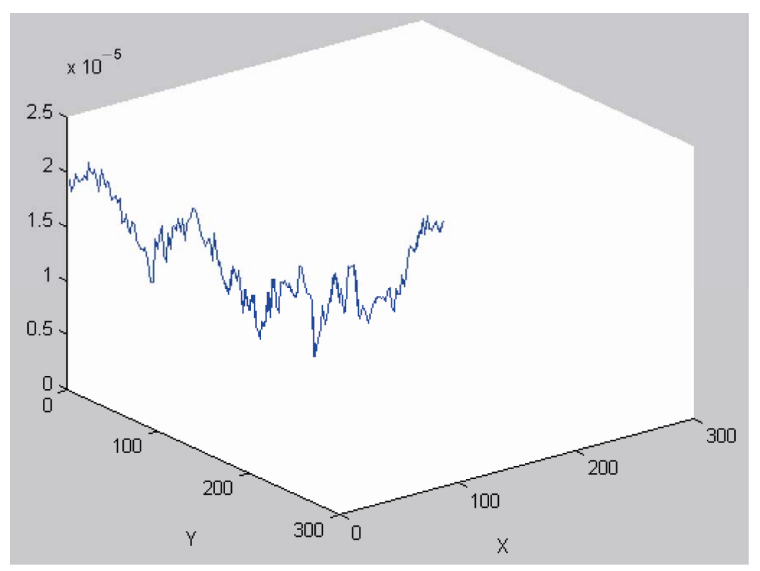

(c)

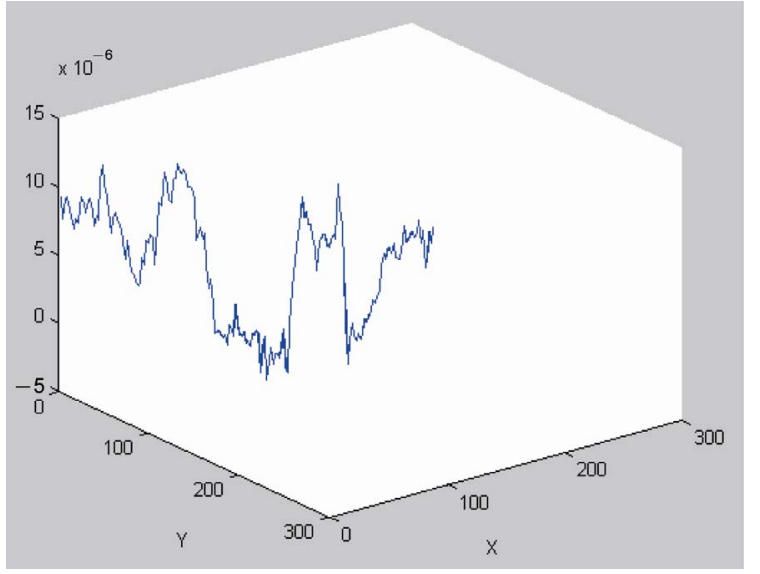

(b)

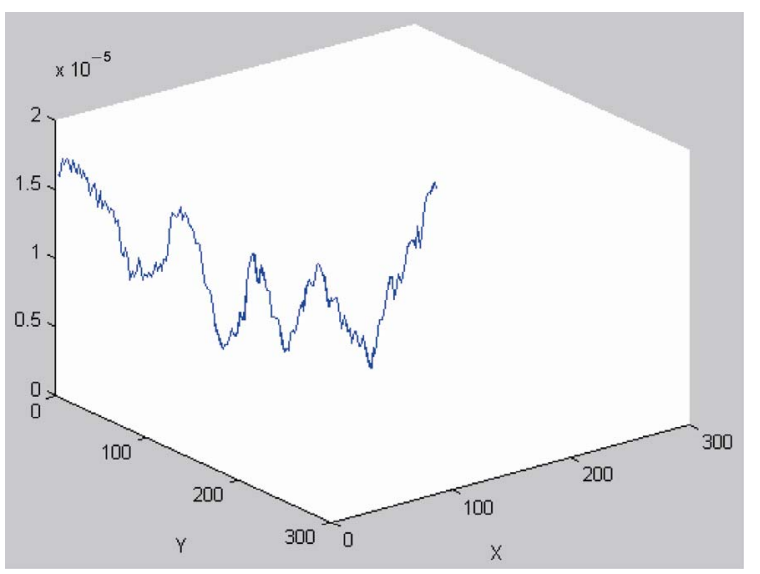

(d) 


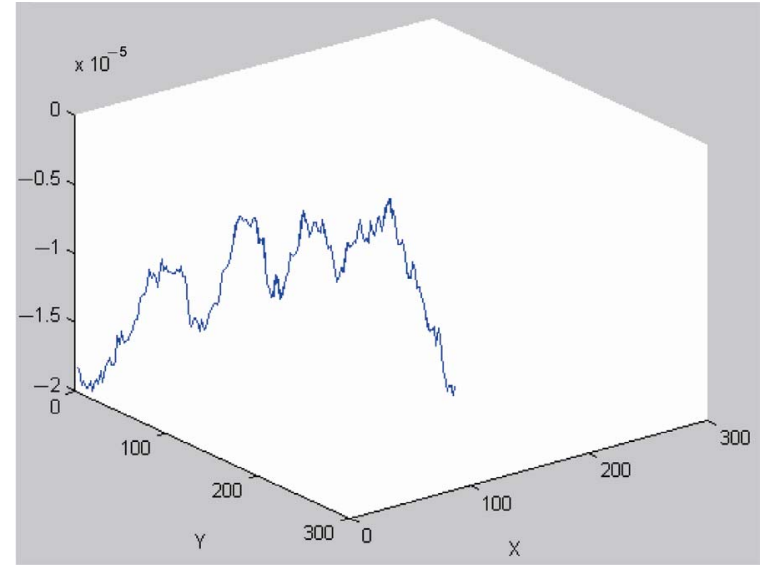

(e)

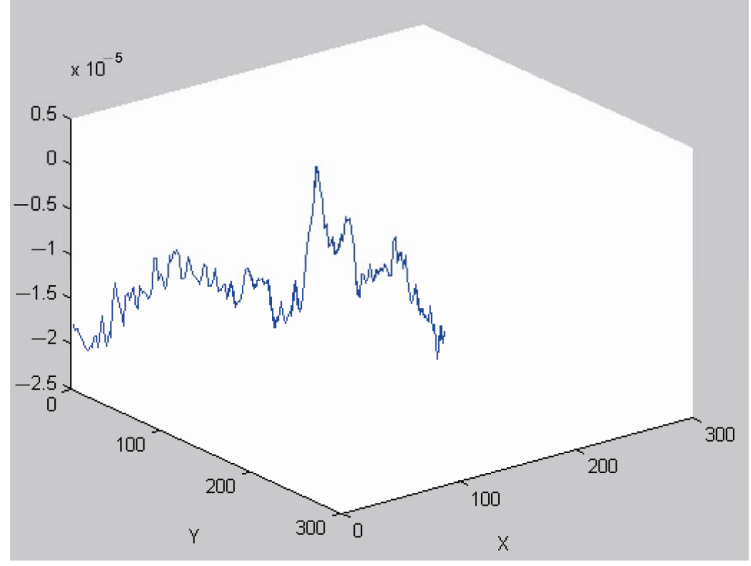

(f)

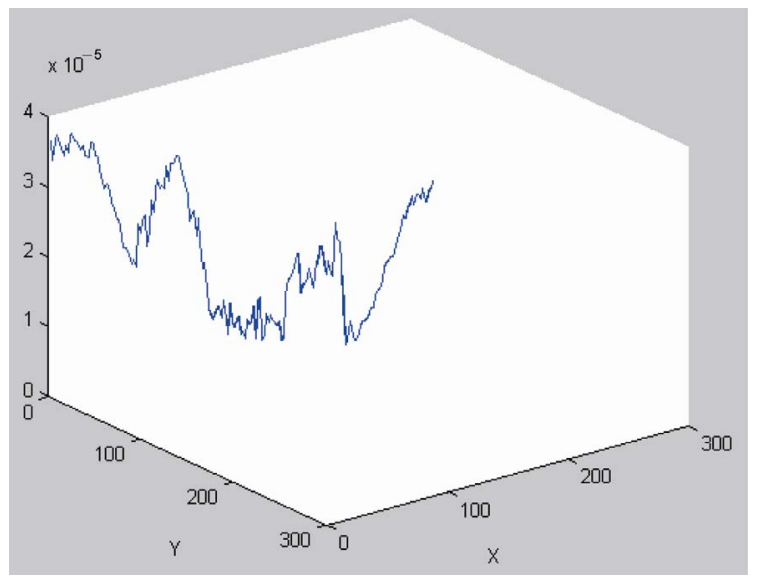

(g)

Figure 6. (a) Image profile of the original image of $H_{1} N_{1}$ virus at slice $x=[12,127,575]$ and slice $y=[1,180,100,100]$; (b) Image profile of the sine FZP reconstructed image at slice $x=[12,127,575]$ and slice $y=[1,180,100,100]$; (c) Image profile of the sine FZP reconstructed image at slice $x=[12,127,575]$ and slice $y=[1,180,100,100]$ using linear amplitude modulation; (d) Image profile of the cosine FZP reconstructed image at slice $x=[12,127,575]$ and slice $y=[1,180,100,100]$; (e) Image profile of the cosine FZP reconstructed image at slice $x=[12,127,575]$ and slice $y=[1,180,100,100]$ using linear amplitude modulation; (f) Image profile of the complex FZP reconstructed image at slice $x=[12,127,575]$ and slice $y=[1,180,100,100]$; (g) Image profile of the complex FZP reconstructed image at slice $x=[12,127,575]$ and slice $y=[1,180,100,100]$ using linear amplitude modulation.

\section{Conclusions}

Firstly, we conclude that the complex FZP hologram gives better resolution for the reconstructed images as compared with the reconstructed images obtained from the sine and cosine FZP holograms.

Secondly, the reconstructed images in case of the sineFZP hologram provided with linearly modulated aperture is better in resolution than the reconstructed images obtained in case of uniform circular pupil. This resolution improvement of the reconstructed holographic images in case of linear pupils is due to the sharp distribution of the PSF obtained in case of linear pupils as compared with the corresponding PSF obtained for circular uniform aperture.

\section{References}

[1] J. W. Goodman and R. W. Lawrence, "Digital Image Formation from Electronically Detected Holograms," Applied Physics Letters, Vol. 11, No. 1, 1967, pp. 77-79. doi:10.1063/1.1755043

[2] A. W. Lohmann and D. P. Paris, "Binary Fraunhoffer Holograms, Generated by Computer," Applied Optics, Vol. 6, No. 10, 1967, pp. 1739-1748. doi:10.1364/AO.6.001739

[3] M. A. Kronrod and L. Yaroslavsky, "Reconstruction of a Hologram with Computer," Soviet Physics: Technical Physics, Vol. 17, No. 2, 1972, pp. 329-332.

[4] K. Nagashima, "Improvement of Images Generated Reconstructed from Computer-Hologram Using an Iterative 
Method," Optics \& Laser Technology, Vol. 18, No. 3, 1986, pp. 157-162. doi:10.1016/0030-3992(86)90076-9

[5] A. M. Hamed, Numerical Speckle Images Formed by Diffusers Using Modulated Conical and Linear Apertures," Journal of Modern Optics, Vol. 56, No. 10, 2009 , pp. 1174-1181. doi:10.1080/09500340902985379

[6] B. B. Gorbatenko, L. A. Maksimova and V. P. Ryabukho, "Reconstruction of the Hologram Structure from a Digitally Recorded Fourier Specklegram," Optics and Spectroscopy, Vol. 106, No. 2, 2009, pp. 281-287. doi:10.1134/S0030400X09020210

[7] N. Takanori and O. Mitsukiyo, et al., "Image Quality Improvement of Digital Holography by Superposition of Reconstructed Images Obtained by Multiple Wavelengths," Applied Optics, Vol. 47, No. 19, 2008, pp. D38-D43. doi:10.1364/AO.47.000D38

[8] J. P. Liu and T. C. Poon, "Two-Step-Only Quadrature Phase Shifting Digital Holography," Optics Letters, Vol. 34, No. 3, 2009, pp. 250-252. doi:10.1364/OL.34.000250

[9] T. C. Poon, "Scanning Holography and Two-Dimensional Image Processing by Acousto-Optic Two-Pupil Synthe- sis," Journal of the Optical Society of America A, Vol. 2, No. 4, 1985, pp. $521-527$.

[10] T. C. Poon, "Optical Scanning Holography with MAT$L A B$," Springer Series in Optical Science, Amazon Co., Dordrecht, 2007.

[11] T. C. Poon, "Three Dimensional Matching by Use of Phase-Only," Journal of Holography and Speckle, Vol. 1 No. 1, 2004, pp. 6-25.

[12] A. W. Lohmann and T. Rhodes, "Two-Pupil Synthesis of Optical Transfer Functions," Applied Optics, Vol. 17, No. 7, 1978, pp. 1141-1150. doi:10.1364/AO.17.001141

[13] T. C. Poon, T. Kim, et al., "Twin-Image Elimination Experiments for Images in Optical Scanning Holography," Optics Letters, Vol. 25, No. 10, 2000, pp. 215-217. doi:10.1364/OL.25.000215

[14] A. M. Hamed, "Image and Super-Resolution in Optical Coherent Microscopes," Optik, Vol. 64, No. 4, 1983, pp. 277-284.

[15] J. W. Goodman, "Introduction to Fourier Optics and Holography," 3rd Edition, Roberts and company publishers, Englewood, 2005. 